\title{
Parámetros demográficos en la población de canes y gatos domésticos en asentamientos humanos del distrito de Ventanilla, Callao-Perú
}

\author{
DEMOGRAPHIC PARAMETERS IN THE POPULATION OF DOGS AND CATS IN HUMAN \\ Settlements of the district of Ventanilla, Callao-Peru \\ Diana Rendón H. ${ }^{1}$, Erika Quintana M. ${ }^{2}$, Ivanna Door M. ${ }^{1}$, Fariva Vicuña A. ${ }^{1}$, \\ Daphne León C. ${ }^{1}$, Néstor Falcón P. ${ }^{1}$
}

\section{RESUMEN}

\begin{abstract}
El estudio tuvo como objetivo determinar indicadores demográficos de la población de canes y gatos domésticos con dueño en asentamientos humanos del distrito de Ventanilla, Callao (Perú). Se realizó un muestreo por conglomerado para seleccionar los grupos poblacionales y un muestreo estratificado y sistemático para seleccionar las viviendas. La información fue recolectada mediante una encuesta. Se calcularon las características demográficas de la tenencia de canes y gatos, y la relación can:persona y gato:persona. El 61.9 y $40.0 \%$ de las viviendas poseían canes y gatos, respectivamente. El número de mascotas por vivienda fue de 1.8 para ambas especies. El número de crías en los canes fue de 4.3 y en gatos de 3.1. La tasa de supervivencia estimada de las crías fue de $56.2 \pm 3.0 \%$ en canes y de $66.4 \pm 3.9 \%$ en gatos. La esperanza de vida estimada fue de $4.21 \pm 0.44$ años en canes y de $2.64 \pm 0.46$ años en gatos. Se estimó que existía 1 can por cada 3.98 personas y 1 gato por cada 5.34 personas. El 23\% de encuestados manifestó mantener canes vagabundos en el exterior de las viviendas. Se espera que la información ofrecida sirva a las autoridades de salud para planificar programas de control poblacional y tenencia responsable de animales de compañía en esta población.
\end{abstract}

Palabras clave: perros; gatos; Ventanilla; mascotas; rabia; zoonosis

${ }^{1}$ Grupo de Salud Pública Veterinaria (SAPUVET-PERÚ), Facultad de Medicina Veterinaria y Zootecnia, Universidad Peruana Cayetano Heredia, Lima, Perú

${ }^{2}$ Municipalidad Distrital de Ventanilla, Callao, Perú

${ }^{3}$ E-mail: diana.rendon@upch.pe

Recibido: 10 de junio de 2017

Aceptado para publicación: 30 de octubre de 2017 
The study aimed to determine demographic indicators of the population of domestic dogs and cats with owner in human settlements in the district of Ventanilla, Callao (Peru). A cluster sampling was performed to select the population groups and a stratified and systematic sampling was used to select the dwellings. The information was collected through a survey. The demographic characteristics of dogs and cats, and the relation dog:person and cat:person was calculated. In total, 61.9 and $40.0 \%$ of the houses owned dogs and cats, respectively. The number of pets per household was 1.8 in both species. The number of offspring in dogs was 4.3 and in cats was 3.1. The estimated survival rate was $56.2 \pm 3.0 \%$ for puppies and $66.4 \pm 3.9 \%$ for kittens. The estimated life expectancy was $4.21 \pm 0.44$ years in dogs and $2.64 \pm 0.46$ years in cats. It was estimated that there were $1 \mathrm{dog}$ per 3.98 persons and 1 cat per 5.34 persons. Twenty-three percent of respondents said they maintained stray dogs outside homes. It is expected that the information provided will serve health authorities to plan population control programs and responsible pet ownership in this population.

Key words: dogs; cats; Ventanilla; pets; rabies; zoonoses

\section{INTRODUCCIÓN}

Los animales de compañía han sido domesticados con el propósito de brindar compañía al hombre, además de ejercer un efecto beneficioso en la prevención y recuperación de la salud física y mental de las personas con quienes conviven o se vinculan (Hugues et al., 2013). Sin embargo, una tenencia no responsable de estos animales conlleva a problemas de salud pública, como propagación de enfermedades zoonóticas, contaminación ambiental por heces y dispersión de basura, accidentes por mordedura o arañazos y accidentes de tránsito, entre otros (Varas et al., 2006; León et al., 2013).

El can doméstico es el principal animal de compañía encontrado en hogares y su población se encuentra en aumento, principalmente en los países en vías de desarrollo, donde la legislación sobre control y tenencia responsable es débil o nula (FAO, 2011). Esta población puede crecer $85 \%$ en 10 años, mientras que el crecimiento de la población humana sería de 23.5\% (Pacheco, 2001).
En América Latina se ha estimado que existen alrededor de 6513000 canes, con una razón can:persona de 1:7.7. Los países con menor proporción de canes por personas son Argentina y Paraguay (1:10), mientras que Chile y Bolivia presentaban una razón can:persona de 1:6.8 y 1:53, respectivamente (OPS, 2003).

Los primeros estudios acerca de estimación de poblaciones de canes y gatos en Lima, Perú, se llevaron a cabo en 1973, donde se registró una población de 300534 canes y 112405 gatos (Málaga, 1973). Información reciente en canes se ha obtenido a partir de estudios realizados en varios distritos de Lima. En el distrito de Comas se encontró que el $60.4 \%$ de hogares tenían canes, estimándose una población de 85934 a partir de una relación 1:5.74 (Soriano et al., 2017). Así mismo, en San Martín de Porres se encontró que los hogares con canes alcanzaban el $58.2 \%$, con una proyección estimada de 82794 canes a partir de una relación de 1:7 (Arauco et al., 2014). Por otro lado, en Huaraz, Ancash, Morales (2015) encontró que el $77.5 \%$ de las viviendas poseía 
canes con una relación de 1:3.4; estimándose, además, un promedio de 4.4 crías por camada y $30 \%$ de mortandad en crías.

El portal web de la municipalidad distrital de Ventanilla, Callao, Perú, indica que el distrito se compone por media docena de urbanizaciones y más de 300 asentamientos humanos, de los cuales solo 200 están reconocidos. En este lugar se desconoce la población de animales de compañía, lo que dificulta la planificación de medidas de intervención en dicha población. Por ello, el objetivo del estudio fue determinar indicadores demográficos para la población de canes y gatos domésticos con dueño en los asentamientos humanos de este distrito.

\section{Materiales Y MéTodos}

El estudio observacional transversal descriptivo se realizó en el distrito de Ventanilla, Callao (Perú) en abril de 2016. El desarrollo de la base de datos y el análisis estadístico se realizó en la Facultad de Medicina Veterinaria y Zootecnia de la Universidad Peruana Cayetano Heredia (FAVEZ$\mathrm{UPCH})$.

La población de estudio fueron las viviendas de 10 asentamientos humanos de Ventanilla. El tamaño de muestra se determinó mediante la fórmula de comprobación de una proporción para poblaciones desconocidas. Las restricciones utilizadas fueron: nivel de confianza del $99 \%$, error máximo admisible del 5\% y proporción referencial del $50 \%$ para datos de familias que poseen una mascota en su vivienda. El tamaño de muestra calculado fue de 664 viviendas.

La selección de las unidades de muestreo fue bietápico. Los 10 asentamientos humanos se seleccionaron mediante un muestreo por conglomerado. Para la elección de las manzanas y viviendas se siguieron los criterios de un muestreo sistemático. Se consideró al $10 \%$ de las manzanas en cada asentamiento humano. Para ello, primero se seleccionaron 10 viviendas por manzana y una de cada dos viviendas por manzana, considerando un promedio de 20 casas por manzana. Cuando no se completaba la muestra con las viviendas de una manzana se tomaba la contigua.

La información se recolectó en un instrumento elaborado y validado en forma conjunta entre profesionales de la FAVEZ-UPCH y la Municipalidad de Ventanilla. El instrumento tomó en cuenta la siguiente información: asentamiento humano de procedencia, número de personas que habitaba la vivienda, tenencia de canes y gatos, información reproductiva de la hembra (camadas y crías paridas) y edad de fallecimiento de la última mascota en ambas especies.

Para el desarrollo de la encuesta se utilizaron dos días del mes de mayo (sábado y domingo) y se contó con el apoyo del personal de la municipalidad y estudiantes de la FAVEZ-UPCH. En total participaron 25 personas, quienes fueron capacitados previamente en el uso de la encuesta. En la vivienda seleccionada, el encuestador solicitó la participación de un adulto y tras la aceptación, se procedió a realizar las preguntas en forma oral y las respuestas eran registradas en el documento.

La información recogida en las encuestas fue transferida a una base de datos en el programa Microsoft Excel. La base fue revisada verificando los datos de cada una de las variables introducidas. El análisis estadístico se desarrolló utilizando el paquete estadístico SPSS 19.0. Se calculó la proporción de viviendas del distrito que poseían canes y gatos, el promedio de canes y gatos por vivienda, la relación can/persona y gato/persona, la tasa de supervivencia de las crías (total de crías nacidas / total de camadas) y la esperanza de vida de los canes y gatos. La información de los encuestados y las características demográficas de las mascotas se resumieron en cuadros de frecuencia. 
Resultados

El estudio recolectó 889 encuestas. El $59.8 \%$ de los encuestados formaban hogares entre 3 y 5 personas, el $30.2 \%$ lo hacían con más de 5 personas y solo el $10 \%$ con 2 o menos personas. Se encontró que el $61.9 \%$ de las viviendas poseían canes y el $40 \%$ tenía gatos. El Cuadro 1 presenta la distribución de la tenencia de mascotas según lugar de muestreo. La mayoría de los encuestados mencionó tener una o dos mascotas (canes o gatos). El Cuadro 2 resume la frecuencia de canes y gatos que se mantenían en los hogares.

El promedio de crías por camada fue de 4.3 y 3.1 para canes y gatos, respectivamente. La tasa de supervivencia estimada de las crías fue de $56.2 \pm 3.0 \%$ en canes y de 66.4 $\pm 3.9 \%$ en gatos (Cuadro 3 ). La esperanza de vida de canes fue calculada en $4.21 \pm 0.44$ años $($ mediana $=3 ;$ moda $=2)$ y la de gatos en $2.64 \pm 0.46$ años (mediana $=2 ;$ moda $=2)$.

El promedio de canes por vivienda fue de 1.8 (1000 canes / 550 viviendas) y de 1.8 en gatos (640 gatos / 356 viviendas). Se encontró una relación de 1 can por cada 3.98 personas (3792 personas / 952 canes) y de un gato por cada 5.34 personas (3261 personas / 611 gatos). El 23\% de encuestados manifestó mantener canes vagabundos en el exterior de las viviendas; por lo general, entre 1 a 2 animales.

\section{Discusión}

La mayoría de viviendas tuvieron mascotas, lo que refleja una interacción animal-humano creciente. En países como Australia, Reino Unido, Alemania y Estados Unidos de América también se observa una creciente tenencia de animales de compañía, reconociendo los efectos beneficiosos que aportan a las personas que conviven con ellos, como son la mejora de la autoestima, reduc- ción del estrés, e incremento del bienestar psicológico, entre otros (Gómez et al., 2007).

El estudio encontró un mayor porcentaje de viviendas que poseían canes en comparación a los que poseían gatos. Esto es similar a lo encontrado en otros estudios como el de Llalla (2012) en Lima, Perú, Caviezel (2014) en Buenos Aires, Argentina, Loza (2014) en Santa Cruz de la Sierra, Bolivia y Canato et al. (2012) en Sao Paulo, Brasil, en los que se reconoce que el perro es la mascota más frecuente en los hogares.

El porcentaje de hogares que poseen canes fue similar a otros estudios realizados en distritos de Lima. Así, Arauco et al. (2014) reporta $58.2 \%$ en hogares de San Martín de Porres y Soriano et al. (2017) con $60.5 \%$ en Comas. La diferencia se encuentra en localidades peruanas que comparten áreas rurales, donde este porcentaje aumenta; por ejemplo, en la ciudad de Huaraz, Ancash fue de 77.5\% (Morales, 2015).

Respecto a la tenencia de gatos, lo observado en los asentamientos humanos de Ventanilla se encuentra por encima de resultados en otras zonas de América del Sur. En Chile, en la provincia de Maipo de la Región Metropolitana se encontró que el $12.6 \%$ de viviendas poseían gatos (Illanes, 2009) y en municipio de Sao Paulo la tenencia alcanzó el 10.6\% de viviendas (Canatto et al., 2012). La razón de la misma estaría asociada a la utilidad de estos animales en la zona, la cual sería la de control biológico de roedores (Sánchez et al., 2016).

El promedio de crías por camada fue superior en canes que en gatos. Morales (2015) encontró un promedio similar de crías por camada en perros (4.4) en la ciudad de Huaraz, Perú, en tanto que Zumpano et al. (2011) reportó un índice de cachorros por hembra de 3.75 y 3.0 en Villa del Parque y Villa Ballester en Buenos Aires, respectivamente. 
Cuadro 1. Distribución de la tenencia de canes y gatos en el distrito de Ventanilla (Callao, Perú), según asentamiento humano de procedencia. Abril 2016

\begin{tabular}{lccccc}
\hline \multirow{2}{*}{ Procedencia } & \multirow{2}{*}{ Hogares } & \multicolumn{2}{c}{$\begin{array}{c}\text { Hogares } \\
\text { con canes }\end{array}$} & \multicolumn{2}{c}{$\begin{array}{c}\text { Hogares } \\
\text { con gatos }\end{array}$} \\
\cline { 3 - 6 } & & $\mathrm{n}$ & $\%$ & $\mathrm{n}$ & $\%$ \\
\hline Ampliación Costa Azul & 68 & 50 & 73.5 & 31 & 45.6 \\
Ampliación B4 & 85 & 52 & 61.2 & 40 & 47.1 \\
José Gabriel Condorcanqui & 56 & 34 & 60.7 & 27 & 48.2 \\
Los Ángeles & 21 & 13 & 61.9 & 12 & 57.1 \\
Los Laureles & 30 & 17 & 56.7 & 10 & 33.3 \\
María Jesús Espinoza & 177 & 100 & 56.5 & 63 & 35.6 \\
Santísima Cruz de Motupe & 63 & 40 & 63.5 & 22 & 34.9 \\
Grupo Residencial D2 & 159 & 81 & 50.9 & 62 & 39 \\
Valle Verde & 101 & 87 & 86.1 & 34 & 33.7 \\
La Victoria & 129 & 76 & 58.9 & 55 & 42.6 \\
\hline Total & 889 & 550 & 61.9 & 356 & 40.0 \\
\hline
\end{tabular}

Cuadro 2. Número de animales de compañía por hogar en el distrito de Ventanilla (Callao, Perú). Abril 2016

\begin{tabular}{ccccc}
\hline Número de animales de & \multicolumn{2}{c}{ Hogares con canes } & \multicolumn{2}{c}{ Hogares con gatos } \\
\cline { 2 - 5 } compañía por hogar & $\mathrm{n}$ & $\%$ & $\mathrm{n}$ & $\%$ \\
\hline 1 & 297 & 54 & 228 & 64 \\
2 & 144 & 26.2 & 65 & 18.3 \\
3 & 66 & 12 & 24 & 6.8 \\
4 & 23 & 4.2 & 16 & 4.5 \\
5 & 6 & 1.1 & 9 & 2.5 \\
$>5$ & 12 & 2.1 & 9 & 2.5 \\
No contestaron & 2 & 0.4 & 5 & 1.4 \\
\hline Total & 550 & 100 & 356 & 100 \\
\hline
\end{tabular}

La tasa de supervivencia fue superior en gatos comparada con la de canes. Esto aparentemente porque los gatos domésticos suelen ser más independientes en cuanto a buscar su propio alimento y bienestar, mientras que los canes son dependientes del cuidado que les proporciona el propietario. No obstante, el nivel de supervivencia de las crías fue menor al de otras zonas del país. En la
Ciudad de Huaraz, Ancash, la supervivencia fue de 70\% (Morales, 2015), mientras que en el distrito de San Martín de Porres llegó a 75.7\% (Arauco et al., 2014). Las diferencias pueden estar asociadas a las condiciones en que se desarrolla la crianza de los animales en el distrito de Ventanilla, donde el nivel de pobreza supera el de las zonas contrastadas (INEI, 2008). 
Cuadro 3. Datos reproductivos de la población de canes y gatos en el distrito de Ventanilla (Callao, Perú). Abril 2016

\begin{tabular}{lcc}
\hline Variable & $\begin{array}{c}\text { Con } \\
\text { canes }\end{array}$ & $\begin{array}{c}\text { Con } \\
\text { gatos }\end{array}$ \\
\hline Encuestados & 122 & 91 \\
Total de camadas & 236 & 186 \\
Total de crías 2015 & 1023 & 575 \\
Crías por camada & 4.3 & 3.1 \\
Crías sobrevivientes & 575 & 382 \\
Supervivencia (\%) & 56.2 & 66.4 \\
\hline
\end{tabular}

La esperanza de vida en canes fue inferior a lo reportado en San Martín de Porres, Lima, donde alcanzó una media de 5.2 años (Arauco et al., 2014). Los altos niveles de pobreza en Ventanilla limitarían los cuidados en la alimentación, sanidad y manejo de los canes, resultando en una mayor mortalidad. Valores próximos al estudio se encontró en San Martín de los Andes, Argentina, donde la esperanza de vida alcanzó a 4.9 años (Brusoni et al., 2007). En cuanto a gatos, la investigación reporta uno de los primeros datos de esta variable para el Perú.

El promedio de canes por vivienda, entre viviendas que poseían estos animales, fue similar a lo encontrado en los distritos limeños de San Martín de Porras y de Comas con resultados de 1.6 y 1.74 , respectivamente (Arauco et al., 2014; Soriano et al., 2017). En el caso de gatos, no hay estudios en el Perú que permitan establecer comparaciones locales; sin embargo, Alves et al. (2005) encontró un promedio de 1.8 gatos por vivienda en el estado de Sao Paulo, Brasil, y Loza (2014) encontró 0.5 gatos por vivienda en Santa Cruz de la Sierra, Bolivia.

En cuanto a la relación can:persona, el resultado de Ventanilla fue similar al obtenido en la ciudad de Huaraz (1:3.4) (Morales, 2015) y en la comunidad Los Jardines de
Manchay de Lima (1:3.9) (Málaga et al., 2014). Una relación menor se registró en el distrito de Comas, Lima con 1:5.7 (Soriano et al., 2017), mientras que en el distrito de San Martín de Porres, Lima, fue de 1 can por cada 7 personas (Arauco et al., 2014).

La relación can:persona es importante para estimar la población de canes y así planificar en forma adecuada las campañas antirrábicas (VAN CAN), que se encuentran inmersas dentro del programa de control, prevención y erradicación de esta zoonosis (León et al., 2014). Se debe de tener en cuenta que la rabia aún representa un problema de salud pública para el Perú, pues se encontraba limitada en Puno y Madre de Dios (Navarro et al., 2007), pero en 2015 se ha extendido al departamento de Arequipa, lo que ha obligado a la Organización Panamericana de la Salud a emitir una alerta epidemiológica exhortando a las autoridades sanitarias de la región a reforzar las acciones necesarias de prevención y control del virus de la rabia, variante canina, para reducir el riesgo de aparición de casos humanos (OPS, 2015).

Según la Organización Mundial de Salud (OMS), para lograr un control y, eventualmente, la erradicación de la rabia, las campañas de vacunación deben ser anuales y alcanzar al menos un $70 \%$ de cobertura (OMS, 2013). Más aún, la «Norma Técnica de Salud para la Prevención y Control de Rabia Humana en el Perú» establece que el número de canes a vacunar en campañas debe ser no menor al $80 \%$ de la población estimada (MINSA, 2017).

En la relación gato:persona se encontró un antecedente local en el estudio realizado por Llalla (2012), quién reportó una relación de 1 gato por cada 18.4 personas a partir de una encuesta desarrollada en colegios de diferentes estratos socioeconómicos. En el mismo estudio, cuando se consideró los resultados de un colegio en San Martín de Porres, esta relación aumentó a 1 gato por cada 11.4 personas. 
Estudios demográficos que incluyen canes y gatos realizados en Latinoamérica encuentran que en el municipio de Taboão da Serra en Sao Paulo, Brasil, la razón animal:persona era de 1:5.14 para canes y de 1:30.6 para gatos (Dias et al., 2004). En los distritos administrativos de Sao Paulo, Brasil, se encontró una relación de 1:4.3 y 1:19.3 para la relación can:persona y gato:persona, respectivamente (Canatto et al., 2012). Así mismo, en Buenos Aires, Argentina, se estimó una relación de 14 canes por cada 100 personas y de 8 gatos por cada 100 personas (Caviezel, 2014), mientras que en Santa Cruz de la Sierra, Bolivia, se estimó que la población de animales de compañía constaba de 390543 canes y 137650 gatos (Loza, 2014). En todos los casos, la cantidad de canes superaba a la de gatos.

Una característica especial se observó en el distrito de Ventanilla. Cerca del $25 \%$ de los encuestados tenían la costumbre de alimentar animales vagabundos, pero no asumían la responsabilidad completa de su tenencia como la de hacerse responsable de su refugio, condición sanitaria y manejo. Dado que estos animales se encontraban en las calles no se aseguraba una adecuada disposición de sus heces, pudiendo representar un reservorio de enfermedades zoonóticas o ser responsables de accidentes por mordedura (León et al., 2013).

Los resultados del estudio representan una línea de base sobre la cual las autoridades del distrito de Ventanilla, Callao, pueden fortalecer los programas de tenencia responsable de animales de compañía a fin de favorecer el desarrollo de comunidades cada vez más saludables. Sin embargo, ello no debe de descuidar la atención de otros factores sociales, culturales y económicos que favorecen la tenencia no responsable de los canes y gatos. Las restricciones en servicios e infraestructuras primordiales para la promoción de la salud (servicios de agua y saneamiento básico) no permiten cambios en las prácticas potencialmente riesgosas asociadas a la tenencia no responsable de mascotas, decreciendo las condiciones para el desarrollo y promoción de una cultura de salud en la población (FAO, 2011).

\section{Conclusiones}

- El 62 y $40 \%$ de viviendas del distrito de Ventanilla tenía canes y gatos como mascotas, con un promedio por vivienda de 1.8 para ambas especies.

- Se encontró la relación de 1 can por cada 3.98 personas y de 1 gato por cada 5.34 personas.

- El promedio de crías en los canes fue de 4.3 y en gatos de 3.1. Se estimó una tasa de supervivencia de crías de $56.2 \pm 3.0 \%$ en canes y de $66.4 \pm 3.9 \%$ en gatos.

- La esperanza de vida estimada fue de $4.2 \pm 0.4$ años en canes y de $2.6 \pm 0.5$ años en gatos.

- $\quad$ E1 23\% de las familias tienen la costumbre de refugiar canes callejeros.

\section{Agradecimientos}

Los autores expresan su agradecimiento por el apoyo recibido en el desarrollo de las encuestas a los promotores de salud de la municipalidad del distrito de Ventanilla: Masías Tolentino, Miguel Guzmán S, Ilsia Torrejón A, Edith Ramos G, Rosa Quiroga J, Dorita Ortiz V, Jorge Chuman L, Juana Huamán E, Carito Chuquipul G, Francisco Veja, José Sánchez, Sonia Huánuco, Alex Monzón M, Liz Llanto; asimismo, al Sr. Freddy Rendón $\mathrm{C}$ y Sr. Richard Vilca R, y a los miembros del Grupo SAPUVET-Perú Almendra Fajardo C, Mariela Gutiérrez R, Milagros Gallegos V, Yhoselin Sheyla S, Estefani Arias, Romi Robles M, Karla Zapata D, Ángel Valdivia Z y Aaron Obregón S.

\section{Literatura CitTAda}

1. Alves M, Matos M, Reichmann M, Dominguez MH. 2005. Dimensionamento da população de cães e gatos 
do interior do Estado de São Paulo. Rev Saúde Pública 39: 891-897. doi: 10.1590/ S0034-89102005000600004

2. Arauco D, Falcón N, León D, Urbina B. 2014. Indicadores demográficos y estimación de la población de perros con dueño en el distrito de San Martín de Porres. Rev Salud Tecno Vet Perú 2: 8392.

3. Brusoni C, Dezzotti A, Fernandez J, Lara J. 2007. Tamaño y estructura de la población canina en San Martín de los Andes (Neuquén). Analecta Vet 27(1): 11-23.

4. Canatto BD, Silva EA, Bernardi F, Mendes MCNC, Paranhos NT, Dias RA. 2012. Caracterização demográfica das populações de cães e gatos supervisionados do município de São Paulo. Arq Bras Med Vet Zootec 64: 1515-1523. doi: 10.1590/S010209352012000600017

5. Caviezel P. 2014. Informe módulo de tenencia responsable y sanidad de perros y gatos. Buenos Aires: Dirección General de Estadísticas y Censos, Ministerio de Hacienda. 26 p.

6. Dias R, Garcia R, Silva D, Amaku M, Ferreira J, Ferreira F. 2004. Estimativa de populações canina e felina domiciliadas em zona urbana do Estado de São Paulo. Rev Saúde Pública 38: 565570. doi: 10.1590/S0034-89102004000400013

7. [FAO] Food and Agriculture Organization of the United Nations. 2011. Dog population management. Banna: FAO. 61 p. [Internet]. Disponible en: http://www.fao.org/3/a-i4081e. pdf

8. Gómez LF, Atehortua CG, Orozco SC. 2007. La influencia de las mascotas en la vida humana. Rev Col Cienc Pec 20: 377-386.

9. Hugues B, Álvarez A, Ledón L, Mendoza M, Castelo L, Domínguez, E, et al. 2013. Efectos beneficiosos de los animales de compañía para los pacientes con enfermedades cardiovasculares. CorSalud 5: 226-229.
10. Illanes J. 2009. Demografía en las poblaciones de perros y gatos en el área rural y urbana de la comuna de la Calera del Tango. Tesis de Médico Veterinario. Chile: Universidad de Chile. 65 p.

11. [INEI] Instituto Nacional de Estadística e Informática. 2008. Censos Nacionales 2007: XI de Población y VI de Vivienda. Lima: INEI. [Internet]. Disponible en: http://censos.inei.gob.pe/ cpv2007/tabulados/

12. León D, Panta S, Yarlequé C, Falcón N. 2013. La convivencia con mascotas en zonas periurbanas: Experiencia en LimaPerú. MV Rev Cien Vet 29(4): 21-25.

13. León D, Soriano JF, Arauco D, Falcón. 2014. Estimación de la población de perros con dueño y perros vagabundos: importancia para la Salud Pública. MV Rev Cien Vet 30(3): 5-10.

14. Llalla H. 2012. Caracterización de la tenencia de mascotas y evaluación de conocimientos, actitudes y prácticas asociadas a riesgos de zoonosis y accidentes entre escolares de educación secundaria en tres distritos de Lima Metropolitana. Tesis de Maestría. Perú: Univ Peruana Cayetano Heredia. 75 p.

15. Loza A. 2014. Caracterización de la población canina y felina en Santa Cruz de la Sierra. Tesis de Médico Veterinario Zootecnista. Santa Cruz de la Sierra: Univ Autónoma «Gabriel Rene Moreno». $57 \mathrm{p}$.

16. Málaga H, Piña J, Denegri A, Stefan $T$, Cedamanos D, Alvarado D, Córdova L, et al. 2014. Priorización de problemas de salud comunitaria en una localidad de Lima Metropolitana en el marco del programa de Municipios Saludables. Rev Per Epidemiol 18(2): 1-4.

17. Málaga H. 1973. Características de la población canina y felina en Lima Metropolitana. Lima: Univ Nacional Mayor de San Marcos. 42 p.

18. [MINSA] Ministerio de Salud. 2017. Norma Técnica de Salud para la Prevención y Control de la Rabia Humana en el Perú. Lima: MINSA. 95 p. [Internet]. Disponible en: https:// 
www.minsa.gob.pe/portalweb/06prevencion/est_san/archivo/2011/ NTS_DE_RABIA.pdf

19. Morales D. 2015. Estimación de la población de canes con dueño en la ciudad de Huaraz, departamento de AncashPerú. Tesis de Médico Veterinario Zootecnista. Lima: Univ Peruana Cayetano Heredia. 28 p.

20. Navarro A, Bustamante J, Sato A. 2007. Situación actual y control de la rabia en el Perú. Rev Peru Med Exp Salud Publica 24: 46-50.

21. [OPS] Organización Panamericana de la Salud. 2003. IX Reunión de directores de los programas nacionales de control de rabia en América Latina. Washington: OPS. $71 \mathrm{p}$.

22. [OPS] Organización Panamericana de la Salud. 2015. Alerta Epidemiológica Rabia. 4 p. [Internet]. Disponible en: http://nubr.co/pEDETL

23. Pacheco A. 2001. La sobrepoblación canina: Un problema con repercusiones para la salud humana. Rev Biomed 12: 290-291.
24. Sánchez G, Sánchez F, Limón D, Gaspar del Ángel B. 2016. El gato: enemigo natural de la fauna urbana nociva. Beneficios a la Salud Pública: intervención comunitaria en una Unidad habitacional. En: XIII Encuentro «Participación de la Mujer en la Ciencia». Guanajuato, México: Centro de Investigaciones en Óptica.

25. Soriano JF, Núñez J, León D, Falcón N. 2017. Estimación de la población de canes con dueño en el distrito de Comas, Lima-Perú. Rev Cien Vet 33(2): 1-10.

26. Varas H, Araneda D, Vergara M, Silva $C$. 2006. La población canina en la Región Metropolitana y sus efectos sobre la salud de las personas. Rev Salud Pública Chile 10(2): 112-114.

27. Zumpano R, Tortosa A, Degregorio OJ. 2011. Estimación del impacto de la esterilización en el índice de crecimiento de la población de caninos. Rev Inv Vet Perú 22: 336-341. doi: 10.15381/rivep.v22i4.333 